\title{
AUTONOMIA DO IDOSO: ESCALA DE AVALIAÇÃO DE CAPACIDADE DE DECISÃO
}

\author{
AUTONOMY OF THE ELDERLY: DECISIONAL CAPACITY ASSESSMENT SCALE
}

Anelise Crippa, Irenio Gomes, Newton Terra

\section{RESUMO}

Objetivou-se, através deste trabalho, criar e validar um instrumento capaz de auxiliar nas demandas relacionadas com a curatela, permitindo uma avaliação uniforme entre os casos. Para tanto, foi desenvolvido um construto com 30 questões, passando pela avaliação de expert's. Surge, assim, a ESCADE - Escala de Avaliação de Capacidade de Decisão, com 18 questões finais, com confiabilidade e validade adequadas, com alfa de Cronbach de 0,814, dividido em quatro domínios: atividade diária, gestão financeira, autogestão e bem-estar. Conclui-se, assim, que se está diante de um questionário auxiliar, que busca instrumentalizar as análises judiciais, com pertinência e eficácia para tal tarefa, sendo ainda pertinente ressaltar a viabilidade de identificar, através dos domínios apresentados, a área de maior dificuldade apresentada pelo possível interdito, o que pode ensejar seu uso, também, na tomada de decisão apoiada.

Descritores: Autonomia. Idoso. Gerontologia.

\section{ABSTRACT}

The objective of this paper was to create and validate an instrument capable of assisting in actions related to interdiction, allowing for a uniform evaluation among cases. For this purpose, a construct with 30 questions was developed and subjected to expert evaluation. Thus, the ESCADE - Decisional Capacity Assessment Scale was created, with 18 final questions, adequate reliability and validity and Cronbach's alpha of 0.814 , being divided into four domains: daily activity, financial management, self-management and well-being. It is concluded, therefore, that this is an auxiliary questionnaire seeking to instrumentalize the judicial analysis with pertinence and effectiveness for this task, and it is also important to highlight the feasibility of identifying, through the presented domains, the area of greatest difficulty presented by a possible interdiction, which may lead to its use for supported decision-making as well.

Descriptors: Autonomy. Elderly. Gerontology. 
Introdução

O aumento da população idosa em nosso país, e a projeção de uma expectativa de crescimento ainda maior, ensejam preocupações que remetem à vários campos do conhecimento, como é o caso das ciências jurídicas. Neste âmbito jurídico, um dos reflexos deste prolongamento da população, com o aparecimento de doenças incapacitantes, acarretam uma incessante tentativa de criação de meios para proteção dos vulneráveis.

Quando se está diante da seara jurídica, uma dessas questões recorrentes ao que tange aos idosos, para buscar essa proteção, é a curatela. Neste procedimento jurídico, a pessoa dita sem autonomia, terá sua capacidade suplementada ao receber um curador. ${ }^{2} \mathrm{~A}$ criação deste instituto (da curatela) baseia-se na proteção de vulneráveis, no entanto, muitas vezes ele pode ser uma tentativa de impedir os atos visando o patromônio e não proteção e cuidado. ${ }^{3}$

$\mathrm{Na}$ instrução do pedido inicial é anexado um laudo médico atestando a incapacidade. No entanto, a verificação desta incapacidade se dá em audiência, por meio de uma entrevista com o magistrado que poderá estar acompanhado de equipe interdisciplinar, havendo, também, laudo de perito nomeado pelo magistrado para verificar se há, de fato, necessidade de interdição. ${ }^{4}$ Assim, os critérios adotados são subjetivos, cabendo ao juiz fazer esta análise e se embasar no laudo médico.

Importantes alterações ocorreram no Código Civil em $2016 .{ }^{5} \mathrm{~A}$ inclusão de moficações oriundas do Estatuto da Pessoa com Deficiência, ${ }^{6}$ que alterou o sujeito que poderia sofrer interdição e incluiu a curatela compartilhada além da tomada de decisão apoiada, são alguns destes pontos que merecem relevência. Ademias, em março de 2016 entrou em vigência o novo Código de Processo Civil7 que se encarregou de também apontar modificações e trazer especificações procedimentais para o processo de interdição.

Até hoje, não há um instrumento almejando de forma ponderável a identificação de mudanças na capacidade decisória. Em função das situações epidemiológicas que se vive atualmente, faz-se necessário um intrumento preciso e adequado, criado e voltado aos idosos, capaz de avaliar a capacidade de tomada de decisão, sendo este o objetivo do presente artigo. Portanto, o objetivo do presente trabalho foi criar e validar um instrumento capaz de auxiliar nas demandas relacionadas com a curatela, permitindo uma avaliação uniforme entre os casos.

Metodologia

O presente trabalho faz parte do projeto PENCE (Programa de Envelhecimento Cerebral). O PENCE é uma parceria da Pontifícia Universidade Católica do Rio Grande do Sul (PUCRS) com a secretaria municipal de saúde de Porto Alegre/RS, na qual visa atingir idosos da Estratégia Saúde da Família de Porto Alegre/RS (ESF) pertencentes a duas gerências da área de abrangência do Hospital São Lucas da PUCRS (Leste e Partenon/Lomba do Pinheiro).

Todas as pessoas que foram convidadas a participar já haviam sido avaliadas quanto a déficit e declínio cognitivo, através do PENCE, bem como doenças neuropsiquiátricas, não contendo nenhuma delas, ou seja, atenderam aos critérios de inclusão. Esta avaliação ocorreu através da aplicação dos instrumentos já utilizados como Escala de Depressão Geriátrica de Yesavage (GDS)8 e Vellori9, através dos agentes comunitários de saúde (ACS) treinados pelo referido Programa.

Participaram da validação deste instrumento pessoas idosas com 60 anos ou mais - com idade média de 69 anos - atendidas pela ESF, totalizando 300 idosos. As coletas se deram nas unidades das ESF e através de visitas domiciliares com a presença do ACS.

Para criação do instrumento para verificação da autonomia, após a formulação das perguntas, foram escolhidos 10 juízes avaliadores (expert) de diferentes profissões ( 2 psiquiatras, 2 neurologistas, 3 profissionais da área do direito 3 profissionais da área da bioética), com o objetivo de verificar a adequação da representação comportamental dos atributos para avaliação de cada uma das questões. Esta avaliação consistiu em ver: clareza, ambiguidade, pertinência e se cada uma das perguntas correspondia a apenas uma resposta. 0 instrumento apresenta quatro grupos importantes: atividades diárias, gestão financeira, autogestão e bem-estar. Após a concordância de $80 \%$ dos juízes, fez com que a pergunta permanecesse no instrumento. Para verificação da confiabilidade e consistência interna foi utilizado o cálculo de alfa de Cronbach.11

Para validação do instrumento foram entrevistadas 10 pessoas para cada pergunta, totalizando 300 indivíduos Por se tratar de um estudo de validação em que o número de participantes deve ser aferido pelo número de questões, multiplicando em 10 pessoas para cada questão, não há que se falar em cálculo de tamanho amostral. Esta etapa ocorreu na própria ESF e nas residências dos participantes. Ao todo participaram 11 ESF's.

Para seleção destes participantes foram utilizados como instrumentos de pesquisa a GDS e a Escala Vellore, aplicados pelos ACS. Todos os ACS participaram de uma capacitação realizada no Hospital São Lucas da PUCRS com a equipe do PENCE antes de iniciar a aplicação dos instrumentos. Como ponte de corte foi utilizado, para o GDS, a pontuação de 0 a 4 e, para o instrumento de triagem cognitiva (Vellore), 14 a 16 para o questionário do idoso e 0 a 2 para o questionário do informante. Nesses idosos selecionados, sem sinais ou sintomas de déficit cognitivo ou depressão, fo aplicada a ESCADE, bem como um questionário com dados sócio-demográficos.

Para comparar a média dos resultados da ESCADE entre as categorias das variáveis sócio-demográficas foram utilizados os testes de Mann-Whitney para os dados dicotômicos e de Kruskall-Wallis para os dados politômicos. 0 programa utilizado foi o SPSS na versão 17.0 .

A investigação teve aprovação do Comitê de Ética em Pesquisa da PUCRS, sob o n 24134013.0.0000.5336, e todos os participantes firmaram, em duas vias, o Termo de Consentimento Livre e Esclarecido, de acordo com a Resolução n 466/12.

\section{RESULTADOS E DISCUSSÃO:}

As questões concernentes à autonomia vêm sendo cada vez mais debatidas no cenário brasileiro atual. Em janeiro de 2016, através do Estatuto da Pessoa com Deficiência (Lei 13.146/2015),6 passou-se a reduzir as categorias que podem sofrer interdição civil, sendo excluídas as pessoas com deficiência.

Agora, tendo a pessoa alguma deficiência que lhe cause um nível inferior de compreensão, e sendo relativamente incapaz poderá ser apoiada através da Tomada de Decisão Apoiada (TDA).10 Desta forma, não haverá mais restrição de sua autonomia, como ocorria antes na interdição.

Continuam sendo passíveis de interdição quando estiver diante de um caso em que a pessoa for pródigo, viciado em tóxico, ébrio habitual ou que por causa transitória ou permanente, não puderem exprimir sua vontade. Assim, estes indivíduos são sujeitos de um processo judicial, tendo como pedido a interdição, que irá designar um curador para suprir a falta de capacidade que se apresenta.

\section{a) Construção do instrumento}

Para construção deste instrumento, aqui denominado Escala de Avaliação de Capacidade de Decisão (ESCADE), foi desenvolvido um questionário o qual se propõe a avaliar a capacidade de decisão/autonomia civil de um indivíduo. Para tanto, foram observadas das especificações de Pasquali e colaboradores ${ }^{11}$ bem como de Streiner e Norman..$^{12}$

Para elaboração do construto foram especificadas e elencadas categorias que seriam a representação comportamental da definição operacional. O passo seguinte foi a expressão dessas categorias em tarefas unitárias e específicas (os itens), chegando-se ao instrumento piloto com 37 questões.

Eles apontaram se cada item elaborado estava se referindo ao fator que se pretende avaliar. A concordância de $80 \%$ dos juízes para cada item fez com que o item permanecesse no construto. Desta forma, chegou-se a 30 questões finais, conforme quadro 1.

Quadro 1: ESCADE inicial, após avaliação dos juízes.

ESCADE Nunca Raramente Às vezes Quase sempre SempreNA*

1. O(A)Sr.(a) se sente apto(a) a tomar decisões?

2. O(A) Sr.(a) toma decisões sobre a sua vida?

3. O(A)Sr.(a) tem vontade de doar de seus bens?

4. O(A)Sr.(a) tem vontade de gastar todo seu dinheiro?

5. O(A) Sr. (a) tem liberdade de escolher onde morar?

6. O(A) Sr.(a) decide quem mora com o Sr.(a)? 
7. O(A)Sr.(a) está desinteressado pela sua vida econômica?

8. O(A) Sr.(a) escolhe o que faz com o seu dinheiro?

9. O(A) Sr.(a) é consultado sobre os planos familiares?

10. O(A) Sr.(a) administra o seu dinheiro?

11. O(A) Sr.(a) administra as contas da casa?

12. O(A)Sr.(a) tem vontade de se separar/divorciar?

13. O(A) Sr.(a) cuida dos seus familiares?

14. O(A) Sr.(a) tem vontade de ajudar o(a)(s) seu(s) (sua[s]) filho(a)(s)?

15. O(A)Sr.(a) já se sentiu desinteressado pela sua vida?

16. O(A) Sr.(a) se sente feliz com a sua vida?

17. O(A) Sr.(a) costuma sair sozinho para passear?

18. O(A) Sr.(a) costuma ir sozinho(a) ao supermercado/farmácia/banco?

19. O(A) Sr.(a) tem liberdade de receber seus amigos em casa?

20. O(A) Sr.(a) tem liberdade de visitar os seus amigos?

21. O(A) Sr.(a) viaja sozinho(a)?

22. O(A) Sr.(a) toma banho sozinho(a)?

23. O(A) Sr.(a) arruma a casa sozinho(a)?

24. O(A) Sr.(a) cozinha?

25. O(A) Sr.(a) escolhe o que come?

26. O(A) Sr.(a) escolhe o que veste?

27. O(A) Sr.(a) auxilia nas tarefas domésticas?

28. O(A) Sr.(a) vota?

29. O(A) Sr.(a) exerce alguma atividade remunerada?

30. O(A) Sr.(a) administra sua vida com autonomia?

*NA = não se aplica.

Para análise semântica dos itens foi feito um brainstorm com grupo de quatro participantes. As respostas foram fornecidas de acordo com a escala de preferências, seguindo Likert, tendo dois dos termos favoráveis, um neutro e dois desfavoráveis (sempre, quase sempre, às vezes, raramente, nunca). Para validação do instrumento foram entrevistadas dez pessoas para cada pergunta, totalizando na versão atual, após o julgamento dos juízes, 300 indivíduos. Esta fase ocorreu na própria ESF e nas residências dos idosos participantes.

A construção de escalas utilizando métodos estatísticos para sua comprovação parece um meio adequado para se buscar, de forma mais palpável, um auxílio aos magistrados no momento da interdição. Algumas enfermidades não deixarão dúvida quanto a sua necessidade de intervenção na capacidade, como no caso de pessoas em coma, porém em outras doenças pode se mostrar necessária e eficaz.

Em consonância com as etapas aqui desenvolvidas, com submissão para juízes avaliadores, tem-se 0 trabalho desenvolvido por Rosa et al, 13 em que os ali chamados de Comitê de Juízes identificaram a equivalência e semântica - uma vez que se tratava de uma tradução e adaptação transcultural de escala -, que os levou a um instrumento final. No referido estudo, ao todo tinham cinco juízes, sendo que eles deveriam ter um dos seguintes critérios: "conhecimento e experiência em adaptação cultural e aplicação de instrumentos de medida, bem como habilidade no reconhecimento de expressões na língua inglesa e desenvoltura com a língua portuguesa".13 Os juízes aqui, avaliaram quatro critérios: clareza, ambiguidade, pertinência e se cada uma das perguntas correspondia a apenas uma resposta.

$O$ teste estatístico para validação de escalas utilizada é o alfa de Cronbach. É através dele que se verifica o quão confiável e válido será fazer estar perguntas para mensurar o intrumento. 14

b) Cálculo do alfa de Crombach e análise fatorial

Com vistas a atingir um padrão de confiabilidade e consistência interna das perguntas elaboradas no construto, foi realizado o teste estatístico alfa de Cronbach. 0 teste mostra quais questões devem ser eliminadas para permitir que se transforme em um instrumento válido, confiável e consistente. Portanto, a partir deste teste, foram excluídas algumas das perguntas, totalizando com 18 questões finais (Quardo 2), pois alguns itens apresentavam baixa consistência dos valores. Observou-se, após a exclusão, um aumento do coeficiente de confiabilidade da escala, passando de 0,618 da versão prévia para 0,814 na versão final.

Quadro 2. Escala de Avaliação de Capacidade de Decisão (ESCADE) após exclusões devido ao cálculo do alfa de Crombach.

ESCALA DE AVALIAÇÃO

DA CAPACIDADE DE DECISÃO

Nunca Raramente

As vezes

Quase sempre SempreNA

1. O(A) Sr.(a) se sente apto(a) a tomar decisões?

2. O(A) Sr.(a) toma decisões sobre a sua vida?

3. O(A) Sr.(a) escolhe o que faz com o seu dinheiro?

4. O(A) Sr.(a) administra o seu dinheiro?

5. O(A) Sr.(a) administra as contas da casa?

6. O(A) Sr.(a) já se sentiu desinteressado pela sua vida?

7. O(A) Sr.(a) se sente feliz com a sua vida?

8. O(A) Sr.(a) costuma sair sozinho para passear?

9. O(A) Sr.(a) costuma ir sozinho(a) ao supermercado/farmácia/banco?

10. O(A) Sr.(a) tem liberdade de receber seus amigos em casa?

11. O(A) Sr.(a) tem liberdade de visitar os seus amigos?

12. O(A) Sr.(a) viaja sozinho(a)?

13. O(A) Sr.(a) toma banho sozinho(a)?

14. O(A) Sr.(a) arruma a casa sozinho(a)?

15. O(A) Sr.(a) cozinha?

16. O(A) Sr.(a) escolhe o que come?

17. O(A) Sr.(a) auxilia nas tarefas domésticas?

18. O(A) Sr.(a) administra sua vida com autonomia?

*NA=não se aplica.

Foram encontrados quatro fatores, divididos em atividade diária (D1), gestão financeira (D2), autogestão (D3) e bem-estar (D4), os quais podem ser vistos no quadro 3.

Quadro 3. Escala de Avaliação de Capacidade de Decisão (ESCADE) de acordo com os domínios. ESCADE

D1 D2 D3

D4

17. O(A) Sr.(a) auxilia nas tarefas domésticas?

14. O(A) Sr(a) arruma a casa sozinho(a)?

15. O(A) Sr.(a) cozinha?

13. O(A) Sr.(a) toma banho sozinho(a)?

4. O(A) Sr.(a) administra o seu dinheiro?

3.O(a) $\mathrm{Sr}(\mathrm{a})$ escolhe o que faz com seu dinheiro?

5. O(A) Sr.(a) administra as contas da casa?

16. O(A) Sr.(a) escolhe o que come?

,889

$\begin{array}{rrr}, 070 & , 730 & , 096 \\ 051 & 480 & 054\end{array}$

$\begin{array}{rrrr}, 940 & 084 & , 093 & , 029\end{array}$

$\begin{array}{lll}, 094 & , 093 & -022\end{array}$

846

$\begin{array}{lll}, 846 & , 126 & -, 025 \\ , 789 & -, 034 & -, 053\end{array}$

, 055

$-, 038$ 
1. O(A)Sr.(a) se sente apto(a) a tomar decisões?

2. O(A) Sr.(a) toma decisões sobre a sua vida?

18. O(A) Sr.(a) administra sua vida com autonomia?

6. O(A)Sr.(a) já se sentiu desinteressado(a) pela sua vida?

9. O(A) Sr.(a) costuma ir sozinho(a) ao

supermercado/farmácia/banco?

11. O(A) Sr.(a) tem liberdade de visitar os seus amigos?

$\begin{array}{rrrr}-, 056 & , 287 & , 811 & -, 181 \\ -, 027 & , 430 & , 758 & -, 148 \\ , 131 & , 463 & , 639 & , 095 \\ -, 054 & -, 172 & , 563 & , 072 \\ & & & \\ , 256 & , 224 & , 520 & , 497 \\ , 177 & -, 062 & , 493 & , 434\end{array}$

$\begin{array}{lrrrr}\text { 10. O(A) Sr.(a) tem liberdade de receber seus amigos em casa? } & -, 053 & -, 043 & -, 085 & , 704 \\ \text { 7. O(A) Sr.(a) se sente feliz com a sua vida? } & -, 111 & -, 052 & -, 068 & , 692 \\ \text { 8. O(A) Sr.(a) costuma sair sozinho(a) para passear? } & , 175 & , 216 & , 492 & , 566 \\ \text { 12. O(A) Sr.(a) pode viajar sozinho(a)? } & , 105 & , 276 & , 430 & , 491\end{array}$

c) Padronização dos valores de referência e influência dos dados sócio-demográficos

Os valores obtidos da escala de avaliação de capacidade de decisão nos idosos em relação ao sexo pode ser verificado na tabela 1. O valor total da autonomia apresentou média de 94,5 para o sexo feminino e 87,8 para 0 masculino. A média tanto para o sexo feminino quanto para o masculino foi maior no domínio bem-estar $(96,6$ sexo feminino; 98,1 sexo masculino) e menor para as mulheres no domínio autogestão $(90,2)$ e para os homens no domínio atividades diárias $(58,6)$. Com relação ao percentil, $75 \%$ dos participantes apresentam um resultado acima de 94 no sexo feminino e acima de 80 no sexo masculino. Com relação aos domínios, mais de $75 \%$ dos participantes apresentaram valores de 100, exceto atividade diária no sexo masculino e autogestão no sexo feminino.

Tabela 1. Valores obtidos da ESCADE na população de acordo com o sexo.

\begin{tabular}{ll}
\hline MEDIDA & $\begin{array}{c}\text { Valor total } \\
\text { da Autonomia D1 - Atividades diárias D2 - Gestão Financeira D3 - Autogestão D4 - Bem- estar }\end{array}$
\end{tabular}

\section{SEXO FEMININO}

\begin{tabular}{|c|c|c|c|c|c|}
\hline $\mathrm{N}$ & 203 & 203 & 203 & 203 & 203 \\
\hline Média & 94,5 & 95,5 & 93,4 & 90,2 & 96,6 \\
\hline Mediana & 100,0 & 100,0 & 100,0 & 100,0 & 100,0 \\
\hline Moda & 100,0 & 100,0 & 100,0 & 100,0 & 100,0 \\
\hline Desvio padrão & 11,0 & 17,6 & 17,0 & 17,7 & 10,6 \\
\hline Mínimo & 45,8 & 0,0 & 25,0 & 0,0 & 12,5 \\
\hline Máximo & 100,0 & 100,0 & 100,0 & 100,0 & 100,0 \\
\hline Percentil 5 & 65,6 & 52,5 & 50,0 & 50,0 & 75,8 \\
\hline 25 & 94,4 & 100,0 & 100,0 & 87,5 & 100,0 \\
\hline 50 & 100,0 & 100,0 & 100,0 & 100,0 & 100,0 \\
\hline 75 & 100,0 & 100,0 & 100,0 & 100,0 & 100,0 \\
\hline \multicolumn{6}{|c|}{ SEXO MASCULINO } \\
\hline $\mathrm{N}$ & 97 & 97 & 97 & 97 & 97 \\
\hline Média & 87,8 & 58,6 & 94,7 & 94,3 & 98,1 \\
\hline Mediana & 88,9 & 62,5 & 100,0 & 100,0 & 100,0 \\
\hline
\end{tabular}

$\begin{array}{lrrrrr}\text { Moda } & 100,0 & 100,0 & 100,0 & 100,0 & 100,0 \\ \text { Desvio padrão } & 12,1 & 38,1 & 18,0 & 13,8 & 6,3 \\ \text { Mínimo } & 41,7 & 0,0 & 0,0 & 50,0 & 58,3 \\ \text { Máximo } & 100,0 & 100,0 & 100,0 & 100,0 & 100,0 \\ \text { Percentil } 5 & 63,9 & 0,0 & 47,5 & 50,0 & 83,3 \\ 25 & 80,6 & 25,0 & 100,0 & 100,0 & 100,0 \\ 50 & 88,9 & 62,5 & 100,0 & 100,0 & 100,0 \\ 75 & 100,0 & 100,0 & 100,0 & 100,0 & 100,0\end{array}$

Na tabela 2 é possível verificar a distribuição das características sócio-demográficas, média e desvio padrão da ESCADE em relação a estas características. Em relação ao sexo, as mulheres apresentaram maior capacidade de tomada de decisão do que os homens. É possível observar nos dados apresentados nesta tabela que quanto mais idosa é a pessoa, menor será a sua capacidade de tomada de decisão. Da mesma forma se verificam os dados de escolaridade em que quanto menor instrução educacional, menor capacidade para decidir terá a pessoa.

Tabela 2. Distribuição das características sócio-demográficas e média e desvio padrão do ESCADE de acordo com estas características.

\begin{tabular}{lrrr}
\hline VARIÁVEL & População N (\%) & ESCADE $m \pm d p$ & $P$ \\
\hline Sexo & & & \\
\hline Feminino & $203(67,7)$ & $94,5 \pm 11,0$ & $<0,001^{*}$ \\
Masculino & $97(32,3)$ & $87,8 \pm 12,1$ & \\
Faixa etária & & & \\
$60-64$ & $89(29,7)$ & $93,0 \pm 10,3$ & $0,002 \#$ \\
$65-69$ & $72(24,0)$ & $94,6 \pm 8,4$ & \\
$70-74$ & $68(22,7)$ & $92,7 \pm 11,8$ & \\
$75-79$ & $42(14,0)$ & $91,8 \pm 12,0$ & \\
80 ou + & $29(9,7)$ & $84,2 \pm 18,5$ & \\
& & & \\
\hline Escolaridade & & $86,6 \pm 18,6$ & $0,035 \#$ \\
& $22(7,3)$ & $91,4 \pm 12,2$ & \\
Analfabeto & $127(42,3)$ & $93,9 \pm 9,3$ & \\
De 1 a 4 anos de estudo (primário) & $102(34,0)$ & $93,8 \pm 10,6$ & \\
De 5 a 8 anos de estudo (ginásio) & $49(16,3)$ & & \\
9 anos de estudo ou mais & & $91,6 \pm 10,5$ & $0,885 \#$ \\
Renda & $22(7,3)$ & $91,6 \pm 13,6$ & \\
Sem remuneração & $126(42,0)$ & $93,0 \pm 10,7$ & \\
Até 1SM & $96(32,0)$ & $92,7 \pm 10,8$ & \\
Até 2SM & $32(10,7)$ & $93,4 \pm 7,9$ & \\
Até 3 SM & $24(8,0)$ & $92,3 \pm 11,8$ & \\
4 SM ou mais & $300(100)$ & & \\
\hline TOTAL & & & \\
& & & \\
\end{tabular}

*Valor de $p$ calculado pelo teste Mann-Whitney.

\#Valor de $\mathrm{p}$ calculado pelo teste Kruskall-Wallis. 
Na associação das variáveis sócio-demográficas com a ESCADE observou-se diferença significativa em relação ao sexo, à faixa etária e à escolaridade. Embora os valores de autonomia médios para o sexo feminino tenham sido maiores que no sexo masculino bem como maior nas faixas etárias mais jovens e de maior escolaridade, a diferença ainda é pequena, mostrando médias elevadas o que corresponde a uma autonomia preservada.

As ausências de outros instrumentos na literatura sobre a mensuração da capacidade de tomada de decisão dificultam a comparação dos resultados encontrados no presente artigo e mostram uma lacuna a ser preenchida quanto à temática.

Como limitação do estudo, ressalta-se que a mesma foi validada em idosos de baixa renda e não foi testada em pessoas nem de renda superior, nem de escolaridade superior.

\section{Considerações Finais}

Ao longo dos próximos anos já terão nossos magistrados um impacto para apontar nas suas decisões com a entrada da TDA em nosso instrumento jurídico. A escala aqui apresentada, que visa englobar qualquer forma de restrição de capacidade, tem por objetivo auxiliar nos julgados. Ela se mostra de fácil utilização e aplicação.

Desta forma, sua validação, em especial para os idosos, mostra-se de suma importância. Pode-se, através dela, ter uma melhor identificação de qual área está sendo afetada e em qual esfera diária será necessário um auxílio.

A escala se mostra com bom nível de fidedignidade e confiabilidade, atráves dos dados mostrados pelo alfa de Cronbach. Apesar disso, pesquisas futuras podem apontar uma forma complementar de detectar alterações na capacidade de tomada de decisão permitindo que este processo ocorra de forma mais objetiva.

\section{Referências}

1.Instuto Brasileiro de Gerografia Estatística (IBGE). Projeção da população do Brasil e das Unidades da Federação. Disponível em: http://www.ibge.gov.br/apps/populacao/projecao/. Acesso em: 13 nov 2016.

2. Madaleno H. Curso de Direito de Família. Rio de Janeiro: Forense, 2013.

3. Tartuce F. Direito Civil: direito de família. Rio de Janeiro: Forense, 2016.

4. Amorim AMA. Manual de direitos das famílias. Curitiba: Juruá, 2016.

5. Brasil. Lei $n^{0}$ Lei $n^{0} 10.406$, de 10 de janeiro de 2002. Institui o Código Civil. Disponível em: http://www.planalto.gov.br/ ccivil_03/leis/2002/L10406.htm. Acesso em 15 nov 2016.

6. Brasil. Lei $n^{0} 13.105$, de 16 de março de 2015. Código de Processo Civil. Disponível em: https://www.planalto.gov.br/ ccivil_03/_ato2015-2018/2015/lei//13105.htm. Acesso em 15 nov 2016.

7. Brasil. Lei $n^{0}$ 13.146, de 6 de julho de 2015. Institui a Lei Brasileira de Inclusão da Pessoa com Deficiência (Estatuto da Pessoa com Deficiência). Disponível em: http://www.planalto.gov.br/ccivil_03/_ato2015-2018/2015/Lei/L13146.htm. Acesso em 15 nov 2016.

8. Yesavage JA, Brink TL. Development and validation of a geriatric depression screening scale: a preliminary report. Journal of Psychiatric Research 1983; 17(1): 37-49.

9. Loureiro F, Finger G, Gomes I. Tradução e adaptação cultural para o português brasileiro do instrumento para rastreio cognitivo Vellore. Pajar 2015;3(2):47-52.

10. RIZZARDO, Arnaldo. Os deficientes e a tomada de decisão apoiada. GenJurídico 2015. Disponível em: http:// genjuridico.com.br/2015/10/21/os-deficientes-e-a-tomada-de-decisao-apoiadal. Acesso em 24 out 2016.

11. PASQUALI, Luiz et al. Instrumentação psicológica: fundamentos e práticas. Porto Alegre: Artmed, 2010.

12. STREINER, David L.; NORMARN, Geoffrey R. Health measurement scales. 4rd ed4. Oxford: Oxford; 2008.

13. Rosa BA, Rodrigues RCM, Gallani MCBJ, Spana TM, Pereira CGS. Estressores em unidade de terapia intensiva: versão brasileira do The enviromental stressor questionaire. Rev Esc Enferm USP 2010; 44(3):627-3.

14. MARTINS, Gilberto de; THEÓPHILO, Carlos Renato. Metodologia da Investigação Científica para Ciências Sociais Aplicadas. São Paulo: Atlas, 2009. 